\title{
Fully Decentralized Cooperative Navigation for Spacecraft
}

\section{Constellations}

\section{Tong Qin, Non-member, Malcolm Macdonald, Non-member, Dong Qiao, Non-member}

Abstract - This paper proposes a method to decentralize the navigation burden, and improve the fault tolerance for a spacecraft constellation. The constellation body reference system is introduced, which is the perifocal frame of one satellite in the constellation. The structure of the proposed navigation method is constructed to enable each spacecraft to estimate its own orbit in this body reference system. This step is essentially the relative orbit determination based on inter-satellite range measurements. Thereafter, the approach to transfer an orbit from the constellation body reference system to inertial reference system is developed. The essential requirements on absolute measurements to realize the coordinate transfer are presented. By dividing the absolute orbit determination into relative orbit determination and coordinate transfer, each navigation sub-system operated in a spacecraft can be independent with others, and the absolute measurements collected by any spacecraft can contribute to the absolute orbit determination of the whole constellation. The proposed method applies to constellations in any geometric configuration. A Walker constellation is taken as an example for numerical simulations. The results show that the proposed method has a lower computation burden compared to an integrated navigation system. With the same type of absolute measurements, the proposed method has higher accuracy and convergence velocity than conventional decentralized algorithms. When a spacecraft occurs with fault, the orbit results of other spacecraft are not affected using the proposed method, which is beyond the ability of conventional methods. 
Index Terms - spacecraft constellation, coordinated navigation, decentralized navigation, relative orbit determination, absolute orbit determination

\section{INTRODUCTION}

Spacecraft cooperation, taking the advantage of inter-satellite measurements, has been widely used to aid navigation performance ${ }^{[1-4]}$. Usually, cooperative navigation relies on a main satellite which processes all measurements collected by other spacecraft and estimates the states of all spacecraft ${ }^{[5-7]}$. This centralized system introduces notable challenges, such as the single-point failure of that one spacecraft on constellation navigation, coupled with an increasing computation burden as the amount of satellites increases. For large-scale spacecraft constellations or similar, a decentralized navigation system is necessary to distribute the navigation task ${ }^{[8-9]}$.

Previous work on decentralized navigation systems focused on developing decentralized estimation algorithms ${ }^{[10-19]}$. The Full Order Extended Kalman Filter (FOEKF) was developed to enable each spacecraft to operate a navigation subsystem and process its own measurements ${ }^{[10-11]}$. Although FOEKF needs no measurement from other spacecraft and hence simplifies the navigation system, it does not efficiently reduce the calculation burden as the states of all spacecraft need to be estimated in each filter. To decrease the state dimension in each filter, the Reduced Order Kalman Filter (ROEKF) was developed by decoupling the observation equation ${ }^{[12-13]}$. Hence, each spacecraft only needs to estimate its own state. However, the optimal states of other spacecraft cannot be obtained in the decoupled observation equation, which limits the estimation accuracy. The Iterative Reduced Order Extended Kalman Filter (IREKF) was developed to compensate the accuracy of ROEKF by involving a procedure of iteration ${ }^{[14-17]}$. Although the accuracy is improved, the iteration process increases the total calculation of the navigation system, which in turn contradicts the original purpose of a decentralized system. 
Systematic contributions to the decentralization of the navigation system have been made from the view of estimation algorithms, but fail to construct a fully decentralized navigation system, in which each spacecraft operates a navigation subsystem totally independent from other spacecraft. Consequently, low computational burden and high navigation accuracy have contradicted each other. Distinct from previous work, this paper focuses on the structure of the navigation system, and develops a fully decentralized cooperative navigation system by dividing the navigation system into two procedures; relative orbit determination (OD), and absolute orientation determination. The constellation body coordinate system (CBCS) is introduced. The relative OD based on inter-satellite range measurements determines the orbits of all spacecraft in CBCS. The absolute orientation determination obtains the orientation of the constellation body system relative to the inertial coordinate system (ICS). Then the absolute orbits are obtained by transforming the orbits from CBCS to the ICS. In the fully decentralized navigation system, each spacecraft operates a navigation subsystem that only determines the relative orbit between itself and its neighboring spacecraft. Thus, the state dimension of each navigation subsystem remains the same as the number of spacecraft increases. The observation equation describing the relationship between the absolute measurements and the CBCS orientation is established, which is then used to solve the CBCS orientation directly.

\section{DECENTRALIZED ESTIMATION METHODS}

\section{A. Navigation models}

Consider a constellation containing $n$ spacecraft. Denote the spacecraft as $S_{i}(i=1,2, \ldots, n)$, the corresponding orbits as $\mathrm{O}_{i}$, and orbit states as $\boldsymbol{X}_{i}$. The states of the integrated navigation system are given by

$$
\boldsymbol{X}=\left[\boldsymbol{X}_{1}, \boldsymbol{X}_{2}, \cdots \boldsymbol{X}_{n}\right]
$$


In two-body dynamics, the state equation is given by

$$
\begin{gathered}
\dot{\boldsymbol{X}}=\left[\dot{\boldsymbol{X}}_{1}, \dot{\boldsymbol{X}}_{2}, \cdots \dot{\boldsymbol{X}}_{n}\right]^{\mathrm{T}}=\left[\boldsymbol{f}\left(\boldsymbol{X}_{1}\right), \boldsymbol{f}\left(\boldsymbol{X}_{2}\right), \cdots \boldsymbol{f}\left(\boldsymbol{X}_{n}\right)\right]^{\mathrm{T}} \\
\boldsymbol{f}\left(\boldsymbol{X}_{i}\right)=\boldsymbol{f}\left(\left[\boldsymbol{r}_{i}, \boldsymbol{v}_{i}\right]\right)=\left[\begin{array}{c}
\boldsymbol{v}_{i} \\
\left.-\mu \frac{\boldsymbol{r}_{i}}{\left|\boldsymbol{r}_{i}\right|^{3}}\right], i=1,2, \cdots, n
\end{array}\right.
\end{gathered}
$$

where $\boldsymbol{r}_{i}, \boldsymbol{v}_{i}$ are position and velocity vectors of spacecraft $\mathrm{S}_{i}, \quad \mu$ is the gravitational constant of the central gravity body. The measurements of the navigation system include the internal measurements such as inter-satellite range measurements and external measurements such as the starlight angles which are the angles between the line-of-sight of the central body and remote stars. Assuming that each spacecraft can communicate and measure the relative range with its neighboring spacecraft, the constellation's internal measurements are given by

$$
\boldsymbol{y}_{i n}=\left[\left|\boldsymbol{r}_{1}-\boldsymbol{r}_{2}\right|,\left|\boldsymbol{r}_{2}-\boldsymbol{r}_{3}\right|, \cdots,\left|\boldsymbol{r}_{n-1}-\boldsymbol{r}_{n}\right|,\left|\boldsymbol{r}_{n}-\boldsymbol{r}_{1}\right|\right]^{T}+\boldsymbol{v}_{i n}
$$

where $v_{i n}$ is the observation noise. Taking the starlight angles as the absolute measurements and assuming spacecraft $\mathrm{S}_{i}$ can observe $n_{i}$ stars, the absolute observation equations are given by

$$
\boldsymbol{y}_{\text {out }}=\left[\left[\frac{\boldsymbol{r}_{1}}{\left|\boldsymbol{r}_{1}\right|} \boldsymbol{s}_{1,1}, \cdots, \frac{\boldsymbol{r}_{1}}{\left|\boldsymbol{r}_{1}\right|} \boldsymbol{s}_{1, n_{i}}\right], \cdots,\left[\frac{\boldsymbol{r}_{n}}{\left|\boldsymbol{r}_{n}\right|} \boldsymbol{s}_{n, 1}, \cdots, \frac{\boldsymbol{r}_{1}}{\left|\boldsymbol{r}_{1}\right|} \boldsymbol{s}_{n, n_{i}}\right]\right]^{T}+\boldsymbol{v}_{\text {out }}
$$

where $\boldsymbol{s}_{n, n_{i}}$ is the direction vector of the $n_{i}{ }^{\text {th }}$ star observed by spacecraft $\mathrm{S}_{n}$. Combining Eqs. (4)(5), the observation equation of the integrated navigation system is given by

$$
\boldsymbol{y}=\left[\boldsymbol{y}_{\text {in }}, \boldsymbol{y}_{\text {out }}\right]^{T}
$$

Using the conventional integrated navigation method, a main spacecraft operates the navigation system consisted of Eqs. (2) and (6). With more spacecraft, the navigation system is faced with increased computational burden. Moreover, if a fault occurs on the main spacecraft, the whole constellation breaks down. 


\section{B. Decentralized estimation methods}

The FOEKF is firstly summarized. Each spacecraft operates the following navigation subsystem.

$$
\left\{\begin{array}{l}
\dot{\boldsymbol{X}}=\left[\dot{\boldsymbol{X}}_{1}, \dot{\boldsymbol{X}}_{2}, \cdots \dot{\boldsymbol{X}}_{n}\right]^{\mathrm{T}}=\left[\boldsymbol{f}\left(\boldsymbol{X}_{1}\right), \boldsymbol{f}\left(\boldsymbol{X}_{2}\right), \cdots \boldsymbol{f}\left(\boldsymbol{X}_{n}\right)\right]^{\mathrm{T}} \\
\boldsymbol{y}_{i}=\left[\left|\boldsymbol{r}_{i}-\boldsymbol{r}_{i-1}\right|,\left[\frac{\boldsymbol{r}_{i}}{\left|\boldsymbol{r}_{i}\right|} \boldsymbol{s}_{i, 1}, \cdots, \frac{\boldsymbol{r}_{i}}{\left|\boldsymbol{r}_{i}\right|} \boldsymbol{s}_{i, n_{i}}\right]\right]+\boldsymbol{v}_{i}
\end{array}\right.
$$

Each navigation subsystem uses only the measurements related to its own spacecraft, but still estimates all states of all spacecraft in the constellation.

An efficient decentralized navigation system requires that each spacecraft operates a subsystem that estimates its own states using only measurements related to itself. The navigation subsystem in spacecraft $S_{i}$ should be given by

$$
\left\{\begin{array}{l}
\dot{X}_{i}=f\left(X_{i}\right)+\boldsymbol{w}_{i} \\
\boldsymbol{y}_{i}=\left[\left|\boldsymbol{r}_{i}-\boldsymbol{r}_{i-1}\right|,\left[\frac{\boldsymbol{r}_{i}}{\left|\boldsymbol{r}_{i}\right|} \boldsymbol{s}_{i, 1}, \cdots, \frac{\boldsymbol{r}_{i}}{\left|\boldsymbol{r}_{i}\right|} \boldsymbol{s}_{i, n_{i}}\right]\right]+\boldsymbol{v}_{i}
\end{array}\right.
$$

Given a prior state $\hat{\boldsymbol{X}}_{i}^{+}\left(t_{k}\right)$ and state error covariance matrix $\boldsymbol{P}_{i}^{+}\left(t_{k}\right)$ at the epoch $t_{k}$, based on the Extended Kalman Filter (EKF), the state update from the epoch $t_{k}$ to $t_{k+1}$ is given by

$$
\begin{gathered}
\hat{\boldsymbol{X}}_{i}^{-}\left(t_{k+1}\right)=\int_{t_{k}}^{t_{k+1}} \boldsymbol{f}\left(\boldsymbol{X}_{i}\right) \mathrm{d} t \\
\boldsymbol{P}_{i}^{-}\left(t_{k+1}\right)=\boldsymbol{\Phi}\left(t_{k+1}, t_{k}\right) \boldsymbol{P}_{i}^{+}\left(t_{k}\right)\left(\boldsymbol{\Phi}\left(t_{k+1}, t_{k}\right)\right)^{T}
\end{gathered}
$$

where $\Phi\left(t_{k+1}, t_{k}\right)$ is the state transformation matrix from $t_{k}$ to $t_{k+1}$. The measurement update is given by

$$
\begin{gathered}
\boldsymbol{K}\left(t_{k+1}\right)=\boldsymbol{P}_{i}^{-}\left(t_{k+1}\right) \boldsymbol{H}_{i}^{-}\left(t_{k+1}\right)\left[\boldsymbol{H}_{i}^{-}\left(t_{k+1}\right) \boldsymbol{P}_{i}^{-}\left(t_{k+1}\right)\left(\boldsymbol{H}_{i}^{-}\left(t_{k+1}\right)\right)^{T}+\boldsymbol{R}_{i}\left(t_{k+1}\right)\right] \\
\hat{\boldsymbol{X}}_{i}^{+}\left(t_{k+1}\right)=\hat{\boldsymbol{X}}_{i}^{-}\left(t_{k+1}\right)+\boldsymbol{K}\left(t_{k+1}\right)\left(\boldsymbol{y}_{i}-\hat{\boldsymbol{y}}_{i}\right)
\end{gathered}
$$




$$
\boldsymbol{P}_{i}^{+}\left(t_{k+1}\right)=\left(\boldsymbol{I}-\boldsymbol{K}\left(t_{k+1}\right) \boldsymbol{H}_{i}\left(t_{k+1}\right)\right) \boldsymbol{P}_{i}^{-}\left(t_{k+1}\right)
$$

where $\boldsymbol{R}_{i}\left(t_{k+1}\right)$ is the observation noise matrix, $\boldsymbol{I}$ is the identity matrix, and the observation Jacobian matrix $\boldsymbol{H}_{i}\left(t_{k+1}\right)$ is given by

$$
\boldsymbol{H}_{i}\left(t_{k+1}\right)=\left.\frac{\partial \boldsymbol{y}_{i}}{\partial \boldsymbol{X}_{i}}\right|_{\boldsymbol{X}_{i}=\hat{\boldsymbol{X}}_{i}^{+}\left(t_{k+1}\right)}
$$

Eqs. (9)-(13) give the procedures of the ROEKF. The states and measurements are decoupled in the decentralized estimations system. However, the accurate states of $\boldsymbol{r}_{i-1}$ is not obtained when calculating $\boldsymbol{H}_{i}\left(t_{k+1}\right)$. Thus, the accuracy of the decentralized navigation is corrupted. To compensate the accuracy, the IREKF is developed by constructing a new measurement noise matrix, given by

$$
\overline{\boldsymbol{R}}_{i}\left(t_{k+1}\right)=\boldsymbol{H}_{i-1}\left(t_{k+1}\right) \boldsymbol{P}_{i-1}^{-}\left(t_{k+1}\right)\left(\boldsymbol{H}_{i-1}\left(t_{k+1}\right)\right)^{T}+\boldsymbol{R}_{i}\left(t_{k+1}\right)
$$

The matrix $\boldsymbol{P}_{i-1}^{-}\left(t_{k+1}\right)$ that describes the error covariance of the coupled states is involved in the measurement noise matrix, which degrades the confidence level of the measurement. Substituting $\boldsymbol{R}_{i}\left(t_{k+1}\right)$ by $\overline{\boldsymbol{R}}_{i}\left(t_{k+1}\right)$ into Eq. (10) and procedure of the IREKF is given by Eqs. (9)(13).

IREKF decreases the computation burden while maintaining navigation accuracy to some extent. But during the estimation, each navigation subsystem is still coupled with the states of other spacecraft. Moreover, in IREKF the state accuracy of spacecraft $S_{i-1}$ influences the state accuracy of spacecraft $\mathrm{S}_{i}$. Specifically, if spacecraft $\mathrm{S}_{i}$ can only measures the inter-satellite range to $S_{i-1}$, the convergence velocity and accuracy of the navigation subsystem in $S_{i}$ is no better than $\mathrm{S}_{i-1}$. In this sense, spacecraft at the end of the communication crosslink in a large- 
scale constellation suffers severs navigation performance degradation. Therefore, the IREKF is not a fully decentralized navigation system, which requires that the measurements and states of each spacecraft are fully independent from others.

\section{ORBIT DETERMINATION IN CONSTELLATION BODY SYSTEM}

\section{A. Constellation body reference system}

Neglecting the orbit perturbation, the whole constellation can be regarded as a net body constituted of many orbits. The CBCS can be described by the perifocal frame. Each orbit has its own perifocal frame, denoted as $\mathrm{R}_{i}$, with its origin located at the mass center of the central body, $x$ axis directed towards the periapse of $\mathrm{O}_{i}, y$ axis in the orbit plane of $\mathrm{O}_{i}$ and directed towards the true anomaly of 90 degrees past the periapse, and $z$ axis perpendicular to the orbit plane of $\mathrm{O}_{i}$ and in the right-handed principle. Every perifocal frame $\mathrm{R}_{i}$ can be regarded as the CBCS. In this paper, the perifocal frame $\mathrm{R}_{1}$ is taken as the CBCS.

\section{B. Orbit determination in constellation body coordinate system}

The first step of the fully decentralized navigation method is to obtain the orbits in the constellation body reference system based on inter-satellite range measurements. According to [20], the semi-major axis, eccentricity, and true anomaly of two orbits can be obtained using only inter-satellite range measurements. In the perifocal frame $\mathrm{R}_{i}$, the position and velocity of $\mathrm{S}_{i}$ can be given by

$$
\begin{gathered}
\boldsymbol{r}_{i}=\left[\frac{a_{i}\left(1-e_{i}^{2}\right)}{1+e_{i} \cos \left(n_{i}\right)} \cos \left(n_{i}\right), \frac{a_{i}\left(1-e_{i}^{2}\right)}{1+e_{i} \cos \left(n_{i}\right)} \sin \left(n_{i}\right), 0\right]^{T} \\
\boldsymbol{v}_{i}=\left[\sqrt{\frac{\mu}{a_{i}\left(1-e_{i}^{2}\right)}} \sin \left(n_{i}\right), \frac{a_{i}\left(1-e_{i}^{2}\right)}{1+e_{i} \cos \left(n_{i}\right)}\left(e_{i}+\cos \left(n_{i}\right)\right), 0\right]^{T}
\end{gathered}
$$

In [20], by projecting two elliptic orbits onto a celestial sphere, and generating two great circles, 
the relative orientation of the two orbits can be expressed in celestial sphere, as shown in Fig.1.

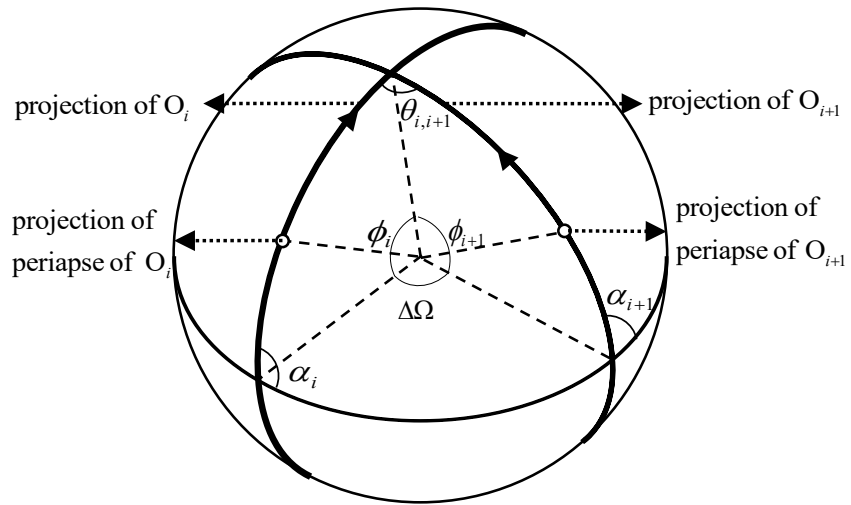

Fig. 1 Relative orientation elements of two elliptic orbits projected onto a starlight sphere

In Fig. $1, \alpha_{i}$ is the inclination angle of orbit $\mathrm{O}_{i}$. The three elements describing the relative orientation are $\theta_{i, i+1}$ which is the angle between the two orbit planes, $\phi_{i}$ which is the angular distance along $\mathrm{O}_{i}$ from the periapse to one of the two intersections of the orbits (this angle is positive in the direction of orbital motion.), and $\phi_{i+1}$ which is the angular distance along $\mathrm{O}_{i+1}$ from the periapse to the same intersection of the orbits. The coordinate transformation matrix from system $\mathrm{R}_{i}$ to $\mathrm{R}_{i+1}$ can be described using the relative orientation elements, given by

$$
\boldsymbol{T}_{i}^{i+1}=\boldsymbol{T}\left(\phi_{i+1}\right) \boldsymbol{T}\left(\theta_{i, i+1}\right) \boldsymbol{T}\left(\phi_{i}\right)
$$

where

$\boldsymbol{T}\left(\phi_{i+1}\right)=\left[\begin{array}{ccc}\cos \left(\phi_{i+1}\right) & -\sin \left(\phi_{i+1}\right) & 0 \\ \sin \left(\phi_{i+1}\right) & \cos \left(\phi_{i+1}\right) & 0 \\ 0 & 0 & 1\end{array}\right], \boldsymbol{T}\left(\theta_{i, i+1}\right)=\left[\begin{array}{ccc}1 & 0 & 0 \\ 0 & \cos \left(\theta_{i, i+1}\right) & \sin \left(\theta_{i, i+1}\right) \\ 0 & -\sin \left(\theta_{i, i+1}\right) & \cos \left(\theta_{i, i+1}\right)\end{array}\right], \boldsymbol{T}\left(\phi_{i}\right)=\left[\begin{array}{ccc}\cos \left(\phi_{i}\right) & \sin \left(\phi_{i}\right) & 0 \\ -\sin \left(\phi_{i}\right) & \cos \left(\phi_{i}\right) & 0 \\ 0 & 0 & 1\end{array}\right]$.

Therefore, the orbit of each spacecraft in its own perifocal frame and the transformation matrix among perifocal frames are obtained via relative OD. The orbit of spacecraft $\mathrm{S}_{i+l}$ in $\mathrm{R}_{i}$ is given by

$$
\begin{gathered}
\boldsymbol{r}_{i+1}^{i}=\boldsymbol{T}_{i}^{i+1} \boldsymbol{r}_{i+1} \\
\boldsymbol{v}_{i+1}^{i}=\boldsymbol{T}_{i}^{i+1} \boldsymbol{v}_{i+1}
\end{gathered}
$$


where $\boldsymbol{r}_{i}^{i+1}$ represents the orbit of spacecraft $\mathrm{S}_{i+1}$ in $\mathrm{R}_{i}$. Likewise, the orbit of each spacecraft in the CBCS is given by

$$
\begin{aligned}
\boldsymbol{r}_{i}^{B} & =\boldsymbol{T}_{1}^{2} \cdots \boldsymbol{T}_{i-1}^{i} \boldsymbol{r}_{i} \\
\boldsymbol{v}_{i}^{B} & =\boldsymbol{T}_{1}^{2} \cdots \boldsymbol{T}_{i-1}^{i} \boldsymbol{v}_{i}
\end{aligned}
$$

Where $\boldsymbol{r}_{i}^{B}$ and $\boldsymbol{v}_{i}^{B}$ are, respectively, the position and velocity of spacecraft $\mathrm{S}_{i}$ in the CBCS. Figure 2 shows the process of obtaining orbits of all spacecraft in the constellation body reference system.

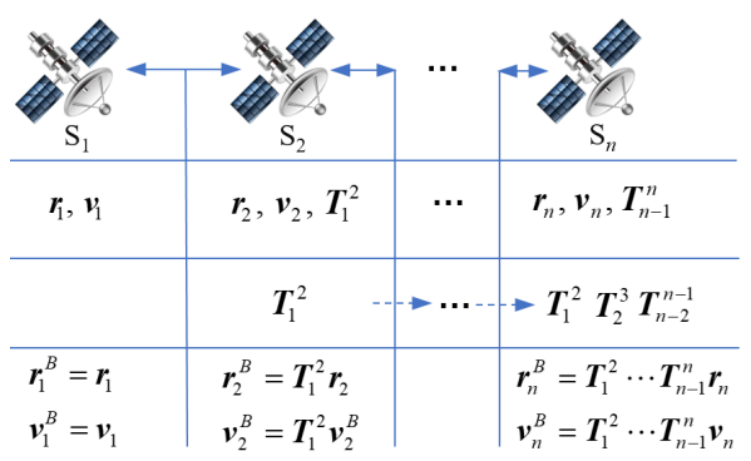

Fig.2 The schematic of the fully decentralized navigation

Under each spacecraft comes the states of the corresponding navigation subsystem. Then the information flow is given, indicating that the coordinates transformation matrix is shared within the constellation. With the state outputs of each spacecraft and the shared transformation matrices, orbits in CBCS are obtained. Note that each navigation subsystem is independent from each other in measurements and states. The shared information is not involved in the estimation process of each navigation subsystem. The state equation of each navigation subsystem is given by

$$
\begin{gathered}
\boldsymbol{X}_{i}=\left[a_{i}, e_{i}, n_{i}, a_{i+1}, e_{i+1}, n_{i+1}, \theta_{i, i+1}, \phi_{i}, \phi_{i+1}\right] \\
\dot{\boldsymbol{X}}_{i}=\boldsymbol{f}\left(\boldsymbol{X}_{i}\right)=\left[0,0, \dot{n}_{i}, 0,0, \dot{n}_{i+1}, 0,0,0\right]^{T}
\end{gathered}
$$




$$
\begin{gathered}
\dot{n}_{1}=\sqrt{\frac{\mu}{a_{1}\left(1-e_{1}^{2}\right)} \frac{\left(1+e_{1} \cos \left(n_{1}\right)\right)^{2}}{a_{1}\left(1-e_{1}^{2}\right)}} \\
\dot{n}_{2}=\sqrt{\frac{\mu}{a_{2}\left(1-e_{2}^{2}\right)} \frac{\left(1+e_{2} \cos \left(n_{2}\right)\right)^{2}}{a_{2}\left(1-e_{2}^{2}\right)}} .
\end{gathered}
$$

The observation equation of each navigation subsystem is given by

$$
\boldsymbol{Y}_{i}=\left\|\boldsymbol{r}_{i}-\boldsymbol{T}_{i}^{i+1} \boldsymbol{r}_{i+1}\right\|
$$

Given the state and measurement equations of relative orbit determination, Unscented Kalman Filter is used to deal with the measurements for state estimation ${ }^{[20]}$. Note that the relative orbit determination can only obtain the orbits in CBCS, and misses the orientation knowledge of CBCS relative to the INS. Therefore, an approach with geometrical meaning to orient CBCS is still needed for absolute orbit determination, which is given in the next section.

\section{ABSOLUTE ORIENTATION DETERMINATION}

\section{A. Orientation determination model}

To obtain the absolute orbits of all spacecraft in the inertial system, the next step is to determine the transformation matrix from CBCS to INS. With a series of absolute measurements, the equation to solve the transformation matrix is given by

$$
\left\{\begin{array}{c}
\boldsymbol{Z}\left(t_{0}\right)=\boldsymbol{h}\left(\boldsymbol{T}_{I}^{B} \boldsymbol{X}^{B}\left(t_{0}\right)\right) \\
\boldsymbol{Z}\left(t_{1}\right)=\boldsymbol{h}\left(\boldsymbol{T}_{I}^{B} \boldsymbol{X}^{B}\left(t_{1}\right)\right) \\
\vdots \\
\boldsymbol{Z}\left(t_{k}\right)=\boldsymbol{h}\left(\boldsymbol{T}_{I}^{B} \boldsymbol{X}^{B}\left(t_{k}\right)\right)
\end{array}\right.
$$

Where $\boldsymbol{Z}\left(t_{k}\right)$ is the measurements at $t_{k}, \boldsymbol{X}^{B}\left(t_{k}\right)$ is the state vector at $t_{k}$ in CBCS, and already obtained via relative OD. Eq. (28) is the general model to solve the transformation matrix $\boldsymbol{T}_{I}^{B}$. The specific solving process of $\boldsymbol{T}_{I}^{B}$ using starlight angles is discussed next. 


\section{B. Orientation determination based on starlight measurements}

In this paper, a kind of classical celestial measurements, starlight angles, are used for CBCS orientation. This angle can be measured by combining a wide field-of-view camera and a startracker, which, respectively, measures the line-of sight of the central body and the remote stars ${ }^{[21-}$ 22]. The measurement schematic is shown in Fig.3.

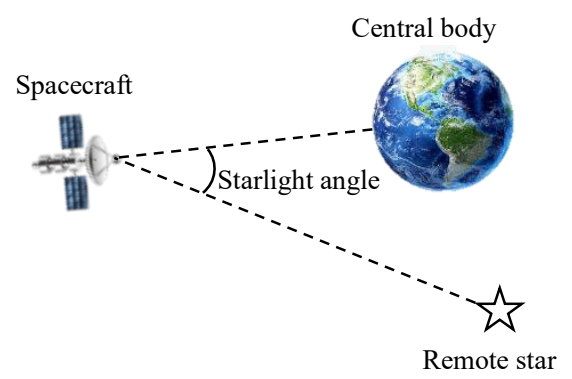

Fig.3 Measurement schematic of starlight angle

The observation model is given as

$$
\alpha_{i, j}=\cos ^{-1}\left(\frac{\left(\boldsymbol{n}_{j}\right)^{T} \cdot \boldsymbol{r}_{i}^{I}}{\left\|\boldsymbol{r}_{i}^{I}\right\|}\right)
$$

where $\alpha_{i, j}$ is the angle between the line-of-sight of the $j^{\text {th }}$ star and the core of the center body measured by spacecraft $\mathrm{S}_{i}, \boldsymbol{n}_{j}$ is the direction vector of the $j^{\text {th }}$ star, $\boldsymbol{r}_{i}^{I}$ is the position vector of $\mathrm{S}_{i}$ in INS. The angle measurement constrains a spacecraft in a circular conical surface whose center axis is along the star direction and half-angle is equal to $\alpha_{i, j}$. Given the rotation matrix from CBCS to INS $\boldsymbol{T}_{I}^{B}$, the observation model in Eq. (29) has the following transformation

$$
\frac{\left(\boldsymbol{n}_{j}\right)^{T} \boldsymbol{T}_{I}^{B} \boldsymbol{r}_{i}^{B}}{\left\|\boldsymbol{r}_{i}^{B}\right\|}=\cos \left(\alpha_{i, j}\right)
$$

where $\boldsymbol{r}_{i}^{B}$ is regarded as a known parameter obtained via relative OD in CBCS. Eq. (30) is essentially an equations set about $\boldsymbol{T}_{I}^{B}$ which has three independent angle parameters. Thus at least 
three observations are required to get a solution, which can be realized by more than three spacecraft or more than three stars. However, due to the nonlinear trigonometry functions, more than one solution can be calculated. Next, the potential solutions of the transformation matrix are discussed.

Case 1: If there is only one star that can be observed, the equations set Eq. (30) has infinite solutions, and the transformation matrix cannot be determined uniquely regardless of the amount of spacecraft.

Provided more than three spacecraft and each measures the starlight angle from the same star. The observation model is given by

$$
\left(\boldsymbol{n}_{j}\right)^{T} \boldsymbol{T}_{I}^{B} \boldsymbol{R}=\boldsymbol{z}
$$

where $\boldsymbol{R}=\left[\boldsymbol{r}_{1}^{B}, \cdots \boldsymbol{r}_{i}^{B}\right], \boldsymbol{z}=\left[\cos \left(\alpha_{1,1}\right), \cdots \cos \left(\alpha_{i, 1}\right)\right]$ and $i \geq 3$. Rotating CBCS along $\boldsymbol{n}_{j}$ by any angle $\theta$, the quaternion expressing the rotation is given by

$$
\boldsymbol{q}=\cos \left(\frac{\theta}{2}\right)+\sin \left(\frac{\theta}{2}\right) \boldsymbol{n}_{j}
$$

The transformation matrix corresponding to the quaternion is given by

$$
\boldsymbol{T}\left(\boldsymbol{n}_{j}\right)=\left[\begin{array}{lll}
q_{0}^{2}+q_{1}^{2}-q_{2}^{2}-q_{3}^{2} & 2\left(q_{0} q_{3}+q_{1} q_{2}\right) & 2\left(q_{1} q_{3}-q_{0} q_{2}\right) \\
2\left(-q_{0} q_{3}+q_{1} q_{2}\right) & q_{0}^{2}-q_{1}^{2}+q_{2}^{2}-q_{3}^{2} & 2\left(q_{2} q_{3}+q_{0} q_{1}\right) \\
2\left(q_{0} q_{2}+q_{1} q_{3}\right) & 2\left(q_{2} q_{3}-q_{0} q_{1}\right) & q_{0}^{2}-q_{1}^{2}-q_{2}^{2}+q_{3}^{2}
\end{array}\right]
$$

where $q_{0}$ is the scalar element of the quaternion, $q_{1}, q_{2}$, and $q_{3}$ are the vector elements of the quaternion. Consider the fact that the rotation around $\boldsymbol{n}_{j}$ does not change the coordinates of $\boldsymbol{n}_{j}$ in the new reference system, given by

$$
\boldsymbol{T}\left(\boldsymbol{n}_{j}\right) \boldsymbol{n}_{j}=\boldsymbol{n}_{j}
$$

Thus, we have 


$$
\left(\boldsymbol{n}_{j}\right)^{T}\left(\boldsymbol{T}\left(\boldsymbol{n}_{j}\right)^{T} \boldsymbol{T}_{I}^{B}\right) \boldsymbol{R}=\boldsymbol{z}
$$

Provided $\tilde{\boldsymbol{T}}_{I}^{B}$ is a special solution for Eq. (31), all solutions are given as

$$
\boldsymbol{T}_{I}^{B}=\boldsymbol{T}\left(\boldsymbol{n}_{j}\right)^{T} \tilde{\boldsymbol{T}}_{I}^{B}
$$

Case 2: If there is only one spacecraft that measures the starlight angle, the equations set Eq. (30) has infinite solutions, and the transformation matrix cannot be determined uniquely regardless of the amount of star.

Provided more than three stars are observed by Spacecraft $\mathrm{S}_{1}$. The observation model is given by

$$
\boldsymbol{N}^{T} \boldsymbol{T}_{I}^{B} \boldsymbol{r}_{i}^{B}=\boldsymbol{z}
$$

where $\boldsymbol{N}=\left[\boldsymbol{n}_{1}, \cdots \boldsymbol{n}_{j}\right], \boldsymbol{z}=\left[\cos \left(\alpha_{1,1}\right), \cdots \cos \left(\alpha_{1, j}\right)\right]$ and $i \geq 3$. Rotating the body system along $\boldsymbol{r}_{i}^{B}$ by any angle $\theta$, the quaternion expressing the rotation is given by

$$
\boldsymbol{q}=\cos \left(\frac{\theta}{2}\right)+\sin \left(\frac{\theta}{2}\right) \boldsymbol{r}_{i}^{B}
$$

The transformation matrix corresponding to the quaternion is given by

$$
\boldsymbol{T}\left(\boldsymbol{r}_{i}^{B}\right)=\left[\begin{array}{lll}
q_{0}^{2}+q_{1}^{2}-q_{2}^{2}-q_{3}^{2} & 2\left(q_{0} q_{3}+q_{1} q_{2}\right) & 2\left(q_{1} q_{3}-q_{0} q_{2}\right) \\
2\left(-q_{0} q_{3}+q_{1} q_{2}\right) & q_{0}^{2}-q_{1}^{2}+q_{2}^{2}-q_{3}^{2} & 2\left(q_{2} q_{3}+q_{0} q_{1}\right) \\
2\left(q_{0} q_{2}+q_{1} q_{3}\right) & 2\left(q_{2} q_{3}-q_{0} q_{1}\right) & q_{0}^{2}-q_{1}^{2}-q_{2}^{2}+q_{3}^{2}
\end{array}\right]
$$

Consider the fact that the rotation around $\boldsymbol{r}_{i}^{B}$ does not change the coordinates of $\boldsymbol{r}_{i}^{B}$ in the new reference system, given by

$$
\boldsymbol{T}\left(\boldsymbol{r}_{i}^{B}\right) \boldsymbol{r}_{i}^{B}=\boldsymbol{r}_{i}^{B} .
$$

Thus, we have

$$
\boldsymbol{N}^{T}\left(\boldsymbol{T}_{I}^{B} \boldsymbol{T}\left(\boldsymbol{r}_{i}^{B}\right)\right) \boldsymbol{r}_{i}^{B}=\boldsymbol{z} .
$$


Provided $\tilde{\boldsymbol{T}}_{I}^{B}$ is a special solution for Eq. (13), all solutions are given as

$$
\boldsymbol{T}_{I}^{B}=\tilde{\boldsymbol{T}}_{I}^{B} \boldsymbol{T}\left(\boldsymbol{r}_{i}^{B}\right)
$$

Case 3: If there are two stars that can be observed and two or more spacecraft that measure the starlight angles, there are no more than two solutions.

Without loss of generality, assume that Spacecraft $\mathrm{S}_{1}$ and $\mathrm{S}_{2}$ both measures two starlight angles. Four measurements can be obtained, given by

$$
\begin{gathered}
{\left[\begin{array}{l}
\boldsymbol{n}_{1}{ }^{T} \\
\boldsymbol{n}_{2}{ }^{T}
\end{array}\right] \boldsymbol{T}_{I}^{B} \boldsymbol{r}_{1}^{B}=\left[\begin{array}{l}
\cos \left(\alpha_{1,1}\right) \\
\cos \left(\alpha_{1,2}\right)
\end{array}\right]} \\
{\left[\begin{array}{l}
\boldsymbol{n}_{1}{ }^{T} \\
\boldsymbol{n}_{2}{ }^{T}
\end{array}\right] \boldsymbol{T}_{I}^{B} \boldsymbol{r}_{2}^{B}=\left[\begin{array}{l}
\cos \left(\alpha_{2,1}\right) \\
\cos \left(\alpha_{2,2}\right)
\end{array}\right]}
\end{gathered}
$$

Firstly, regard $\boldsymbol{T}_{I}^{B} \boldsymbol{r}_{1}^{B}$ and $\boldsymbol{T}_{I}^{B} \boldsymbol{r}_{2}^{B}$ as the vectors to be solved. Eqs. (43) and (44) are both linear equations that have three unknowns and two constraints. From the view of observation geometry, the solutions of the equation sets are the intersection lines of two circular conical surfaces with the star vector as their central axes, shown in Fig.4. There is at least one and at most two intersection lines, which means there is no more than two solutions of $\boldsymbol{T}_{I}^{B} \boldsymbol{r}_{1}^{B}$. Likewise, $\boldsymbol{T}_{I}^{B} \boldsymbol{r}_{2}^{B}$ also has no more than two solutions. Since the transformation matrix can be determined uniquely if two vectors are known, and their coordinates in the transformed system, at most four combinations of $\boldsymbol{T}_{I}^{B} \boldsymbol{r}_{1}^{B}$ and $\boldsymbol{T}_{I}^{B} \boldsymbol{r}_{2}^{B}$ gives four potential solutions. However, a further constraint

$$
\left(\boldsymbol{r}_{1}^{B}\right)^{T} \boldsymbol{r}_{2}^{B}=\left(\boldsymbol{r}_{1}^{I}\right)^{T} \boldsymbol{r}_{2}^{I}
$$

means the coordinate transformation does not change the angle between two vectors. The intersection lines of two conical surfaces are symmetric about the surface formed of the two star vectors. Four intersection lines can only generate two angles. The constraint in Eq. (45) eliminates 
one angle and decreases the amount of solutions from four to two. The geometrical schematic is given in Fig. 4. Fig.4(a) shows the situation where two spacecraft are located in the surface formed by the two star vectors and two conical surfaces are tangent with each other. In this case, only one combination of $\boldsymbol{T}_{I}^{B} \boldsymbol{r}_{1}^{B}$ and $\boldsymbol{T}_{I}^{B} \boldsymbol{r}_{2}^{B}$ can be obtained and thus the solution for $\boldsymbol{T}_{I}^{B}$ is unique. Fig.4(b) is a general situation where two pairs of conical surfaces are intersected with each other and two symmetric solutions are obtained. One combination is depicted in red lines and the other is on the opposite side of the surface formed by the two star vectors.

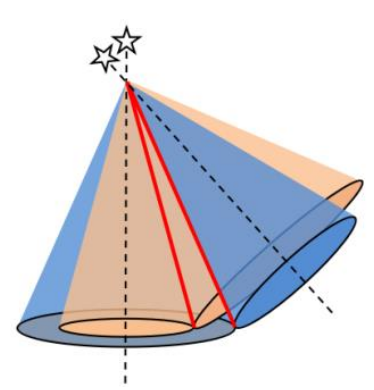

(a) Two intersection lines

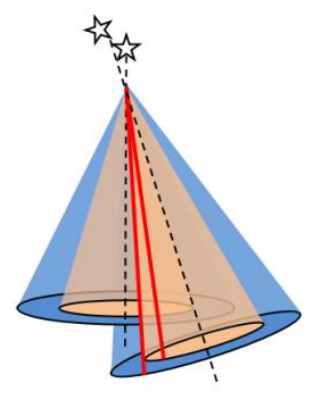

(b) Four intersection lines

Fig.4 Potential observation geometries constructed by starlight angle measurements

Case 4: If there three or more non-coplanar stars can be observed and two or more spacecraft measure the starlight angles, there is only one solution for the transformation matrix.

When there are three non-coplanar stars and two spacecraft, the observation equations are given by

$$
\begin{aligned}
& {\left[\begin{array}{l}
\boldsymbol{n}_{1}^{T} \\
\boldsymbol{n}_{2}{ }^{T} \\
\boldsymbol{n}_{3}{ }^{T}
\end{array}\right] \boldsymbol{T}_{I}^{B} \boldsymbol{r}_{1}^{B}=\left[\begin{array}{l}
\cos \left(\alpha_{1,1}\right) \\
\cos \left(\alpha_{1,2}\right) \\
\cos \left(\alpha_{1,3}\right)
\end{array}\right]} \\
& {\left[\begin{array}{l}
\boldsymbol{n}_{1}{ }^{T} \\
\boldsymbol{n}_{2}{ }^{T} \\
\boldsymbol{n}_{3}{ }^{T}
\end{array}\right] \boldsymbol{T}_{I}^{B} \boldsymbol{r}_{2}^{B}=\left[\begin{array}{l}
\cos \left(\alpha_{2,1}\right) \\
\cos \left(\alpha_{2,2}\right) \\
\cos \left(\alpha_{2,3}\right)
\end{array}\right]}
\end{aligned}
$$


Eqs. (46) and (47) are both linear equations about $\boldsymbol{T}_{I}^{B} \boldsymbol{r}_{1}^{B}$ and $\boldsymbol{T}_{I}^{B} \boldsymbol{r}_{2}^{B}$. Therefore, a unique solution can be obtained for $\boldsymbol{T}_{I}^{B} \boldsymbol{r}_{1}^{B}$ and $\boldsymbol{T}_{I}^{B} \boldsymbol{r}_{2}^{B}$ as long as the coefficient matrix are nonsingular, given by

$$
\operatorname{det}\left(\left[\boldsymbol{n}_{1}, \boldsymbol{n}_{2}, \boldsymbol{n}_{3}\right]\right)=\boldsymbol{n}_{1}\left(\boldsymbol{n}_{2} \times \boldsymbol{n}_{3}\right) \neq 0
$$

The non-singularity requires that the star vectors are non-coplanar. Therefore, if two spacecraft observe starlight angles of three non-coplanar stars, the transformation matrix can be uniquely determined. With more measurements, the transformations matrix is also uniquely determined.

The above theorems analyze the uniqueness of the transformation matrix with measurements at one epoch and without a priori knowledge. Considering the movement of the spacecraft, one spacecraft is enough to determine the transformation matrix if sequential measurements are collected. Furthermore, if an a priori knowledge is given, the symmetric solution in theorem 3 can be eliminated and the transformation matrix is then determined uniquely.

Although, theoretically, three stars lead to a unique rotation angle, the star distribution is related to the solution accuracy when considering observation noise. From the view of mathematics, the non-singularity of the matrix guarantees the uniqueness of solutions, and further, the value of the determinant reflects the accuracy of solutions when adding disturbance to the observation vectors. The maximum of the determinant is one, and occurs when the three vectors are perpendicular to each other. Therefore, to obtain a high navigation accuracy, ideally, the line-of-sight of stars should be perpendicular to each other. Note that, except for starlight angles, other absolute measurements, such as radio ranging or velocity from ground station, X-ray pulsar measurement, and the line-ofsight measurement of the central body, can also be used to determine the absolute orientation of CBCS. 


\section{NUMERICAL SIMULATION}

Numerical simulations are performed to validate the performance of the fully decentralized navigation method in convergence, computation burden, fault tolerance, and navigation accuracy. Comparison to the integrated navigation and IREKF is also conducted. A constellation with 6 spacecraft in elliptic orbits is considered. The orbit elements are listed in Table 1 where $i=1,2,--$, 6. Spacecraft $\mathrm{S}_{1} \sim \mathrm{S}_{6}$ have the same semi-major axis, eccentricity, inclination, and argument of periapse. The longitude of ascending node and true anomaly are both at 60 -degree intervals. Note that the fully decentralized navigation method applies to constellations in any geometric configuration. This section only focuses on an elliptic Walker constellation. The constellation is shown in Fig. 5.

Table 1 Orbit elements of an elliptic constellation

\begin{tabular}{ccccccc}
\hline \hline & $a / \mathrm{km}$ & $e$ & $\alpha / \mathrm{deg}$ & $\omega / \mathrm{deg}$ & $\Omega / \mathrm{deg}$ & $n / \mathrm{deg}$ \\
\hline $\mathrm{S}_{i}(\mathrm{i}=1,2, \ldots, 6)$ & 26560 & 0.3 & 60 & 10 & $60 i-10$ & $60 i-10$ \\
\hline
\end{tabular}

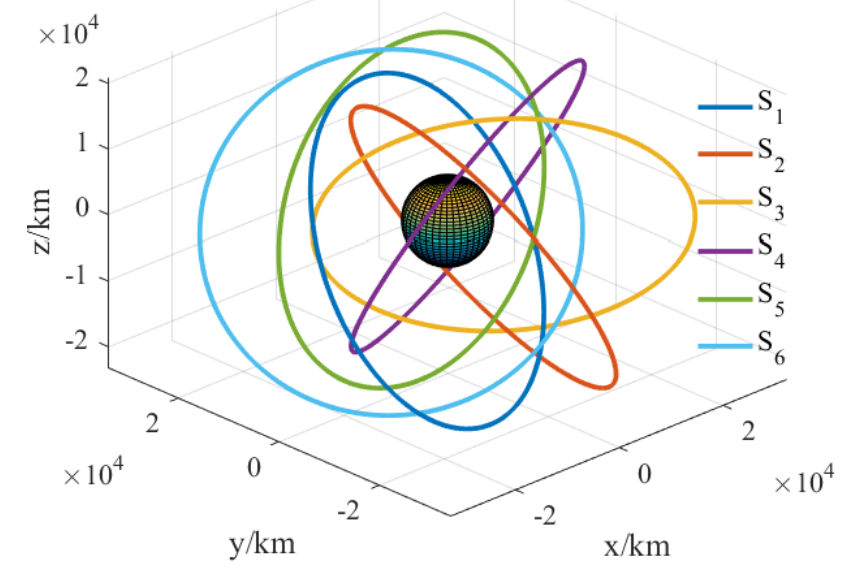

Fig.5. Configuration of an elliptic constellation

\section{A. Convergence}

The convergence performance of the fully decentralized navigation method includes the convergence performance in CBCS and in ICS. Assuming each spacecraft in Table 1 has initial 
tri-axial position errors of $10 \mathrm{~km}$, the orbit determination errors in CBCS over time, as the intersatellite range measurements are made is shown in Fig. 6. It is shown in Fig. 6 that internal measurements are enough to estimate the states of all spacecraft in CBCS. As each spacecraft is independent from each other, the convergence speeds are all similar. Under the same absolute observation conditions, the fully decentralized navigation should have the similar convergence performance to the integrated navigation. In this part, several cases are studied to compare the performance in exploiting the absolute information.
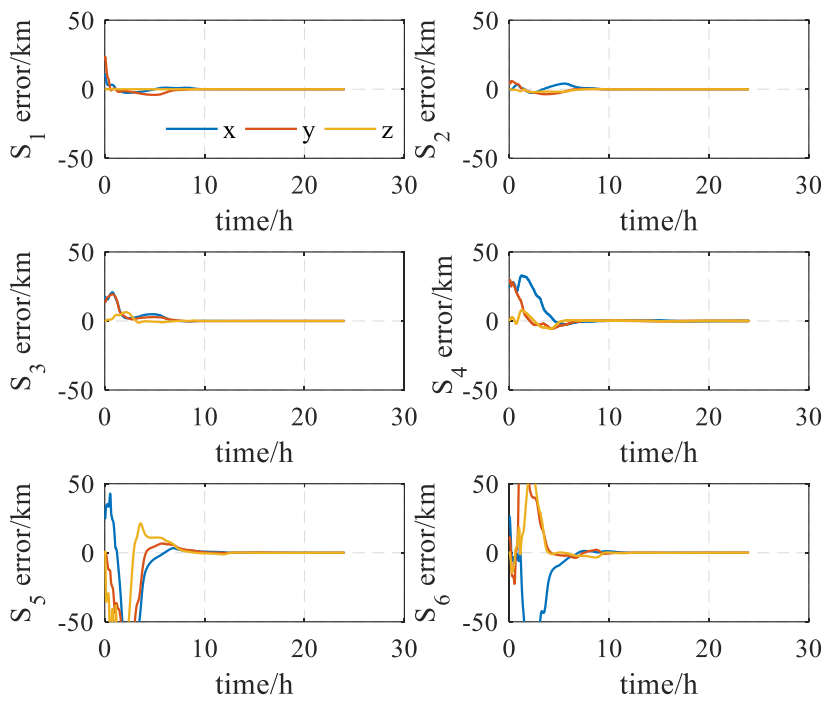

Fig 6. Orbit determination errors in CBCS using only inter-satellite range measurements

\section{Case 1: Spacecraft $S_{1}$ observes one star}

To validate the conditions of calculating the absolute orientation of $\mathrm{CBCS}$, an extreme case where only one spacecraft measures one starlight angle is studied. The direction vector of the star in the inertial reference system is $[1,0,0]$. The navigation errors of integrated navigation, IREKF and fully decentralized navigation are shown in Fig. 7.

It is shown in Fig. 7 that the orbit errors do not converge, indicating that the absolute measurements are not enough to estimate the coordinates transformation matrix even though the orbits in CBCS are determined. Since it is assumed only the star with direction vector $[1,0,0]$ is 
observed, only the x-axis position error converges, supporting the conclusion in section 4 . The navigation errors using IREKF and integrated navigation, shown in Fig. 7(b) and 7(c), have the similar tendency. Therefore, one starlight angle measurement from one spacecraft is not enough to determine the absolute orbits of all spacecraft in the constellation. The state uncertainties, represented by the standard deviation of the state errors, are shown in Table 2 to quantify the navigation performance of the integrated navigation (annotated as IN), fully decentralized navigation (annotated as FDN), and IREKF. The tri-axial position uncertainties of the six spacecraft are averaged, and the initial and final uncertainties are both conveyed.
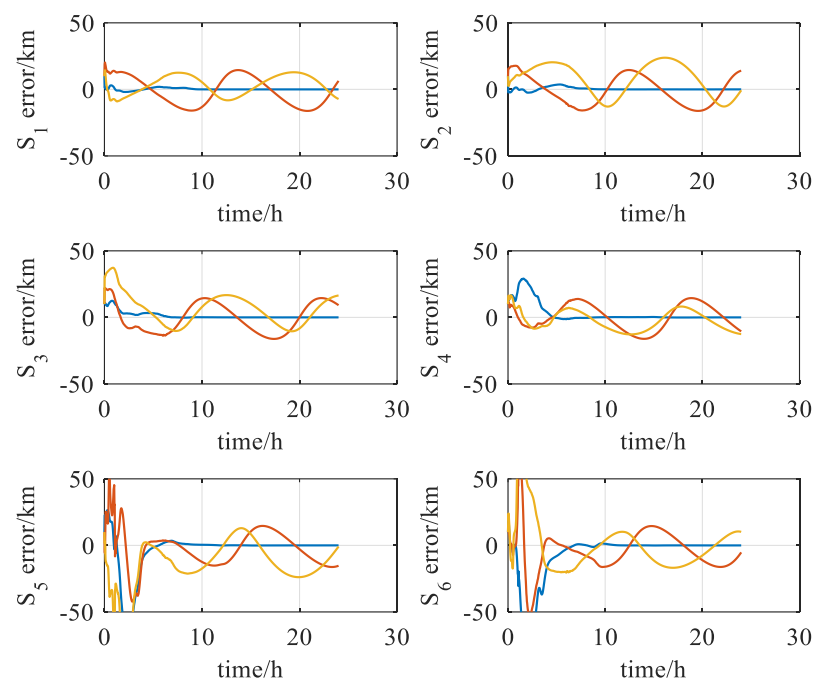

(a) fully decentralized navigation
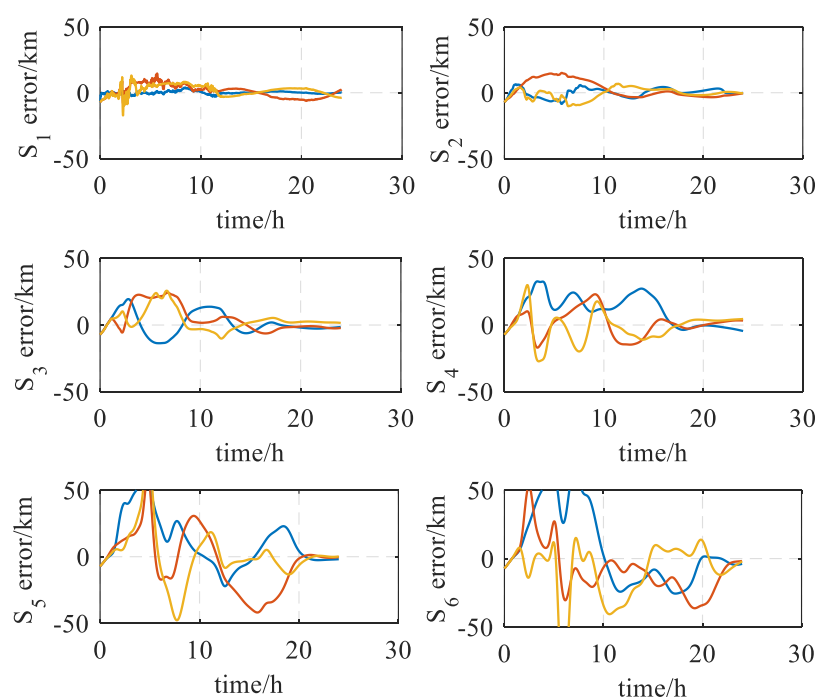

(b) IREKF 

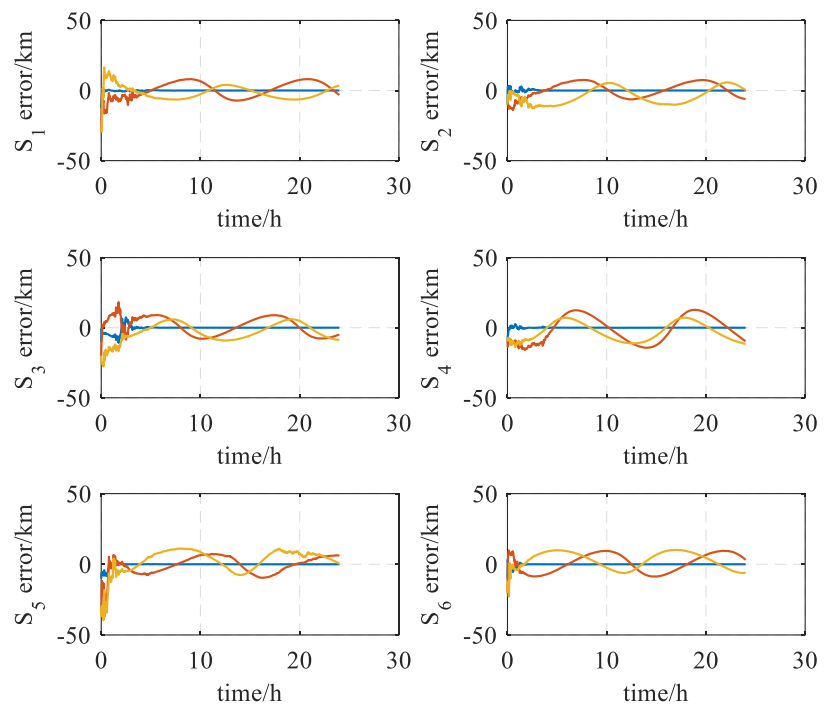

(c) Integrated navigation

Fig. 7 Orbit determination errors when $S_{1}$ observes one star with the direction $[1,0,0]$ using fully decentralized navigation method, integrated navigation method and IREKF respectively

Table 2 Average state uncertainty when $\mathrm{S}_{1}$ observes one star with the direction $[1,0,0]$

\begin{tabular}{ccccc}
\hline \multicolumn{2}{c}{ Methods } & IN & FND & IREKF \\
\hline $\mathrm{X} /$ & initial & 10 & 10 & 10 \\
$\mathrm{~km}$ & final & 0.010 & 0.011 & 2.825 \\
$\mathrm{Y} /$ & initial & 10 & 10 & 10 \\
$\mathrm{~km}$ & final & 12.212 & 11.018 & 10.962 \\
$\mathrm{Z} /$ & initial & 10 & 10 & 10 \\
$\mathrm{~km}$ & final & 11.184 & 12.976 & 10.115 \\
\hline
\end{tabular}

\section{Case2: Each spacecraft observes one starlight angle}

In this case, it is assumed that each spacecraft is capable of measuring one starlight angle, and different spacecraft observes different star. The navigation errors using different navigation methods are shown in Fig. 8. The tri-axial state uncertainties are listed in Table 3. The navigation errors do not converge when each spacecraft estimates its own orbit using only starlight angle measurements, illustrating that the spacecraft cannot determine its orbit without the inter-satellite measurements in this case. Under the same measurement conditions, the orbit determination errors 
using IREKF do not converge either. The increased observation noise matrix in Eq. (15) actually decreases the weight of inter-satellite range measurements in the estimation. Without the effect of inter-satellite range measurements, this case is equivalent to the situation where each spacecraft is using only the absolute measurement of one starlight angle to estimate its own orbit.

The integrated navigation possesses the shortest convergence time. That is because the integrated navigation system is an optimal estimation system which involves all states and measurements in a navigation filter. The navigation errors of the proposed fully decentralized navigation method also converge. The convergence rate depends mainly on the convergence rate of the states in CBCS. Only by obtaining the orbits in CBCS can the absolute orientation of CBCS be determined accurately. The fully decentralized navigation method uses inter-satellite range measurements to estimate the orbits in CBCS, and all absolute measurements collected by each spacecraft is gathered to solve the transformation matrix from CBCS to ICS. In this way, the distributed absolute measurements contribute to obtaining the orbits of every spacecraft, and the spacecraft cooperation is realized.
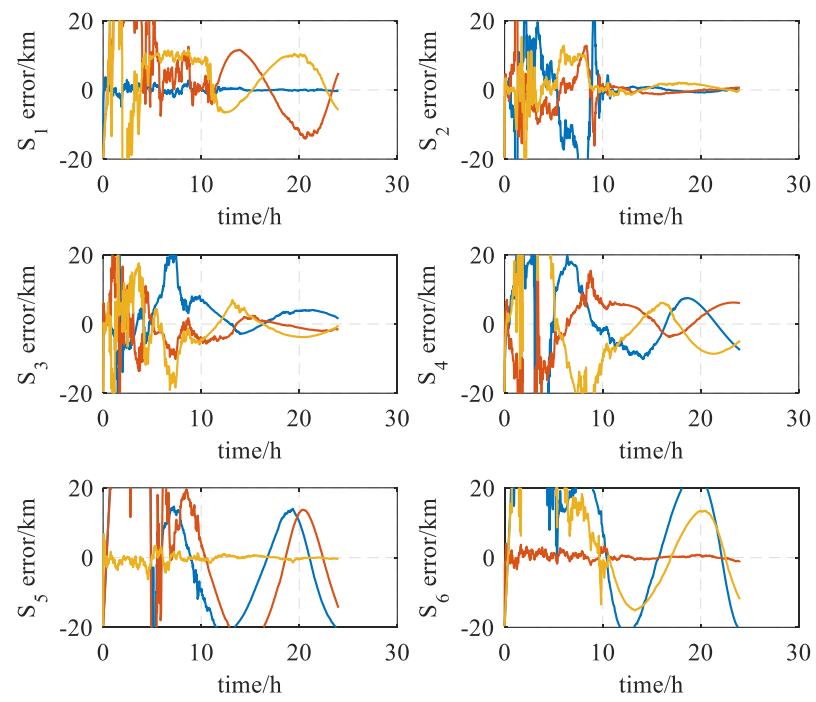

(a) Each spacecraft estimate its own orbits using only starlight angle measurements 

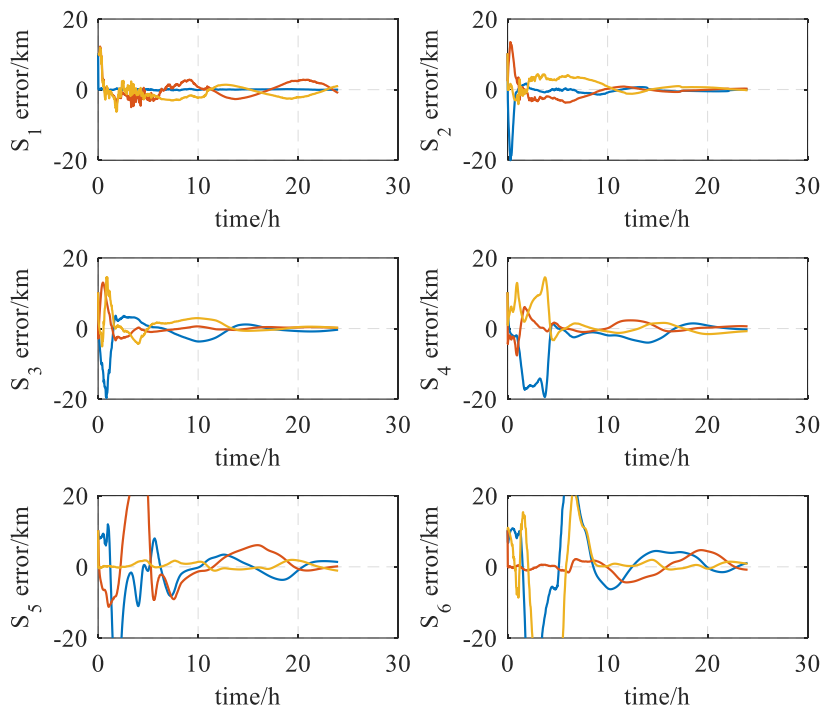

(b) IREKF
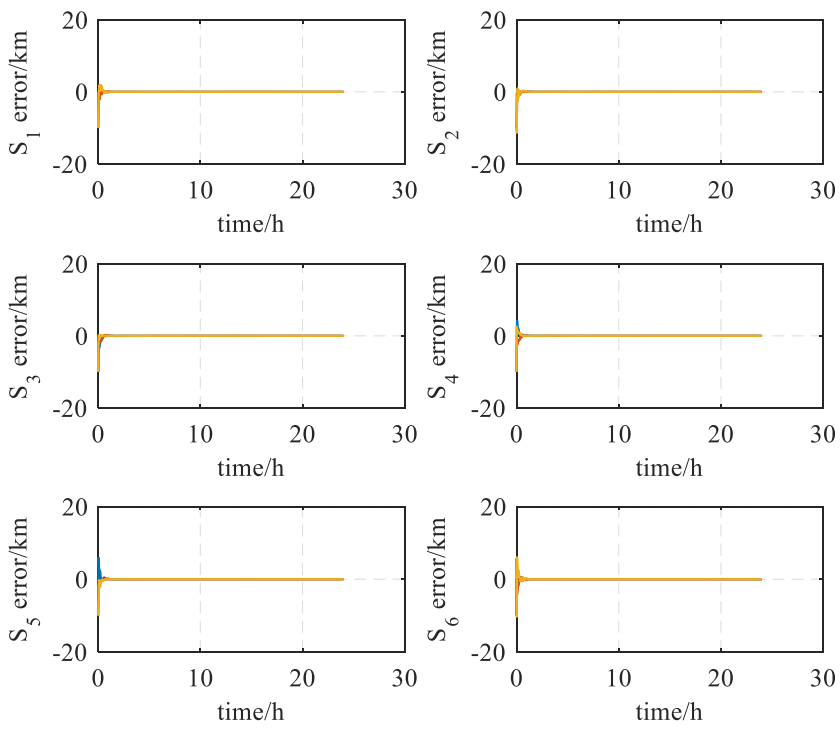

(c) Integrated navigation method 

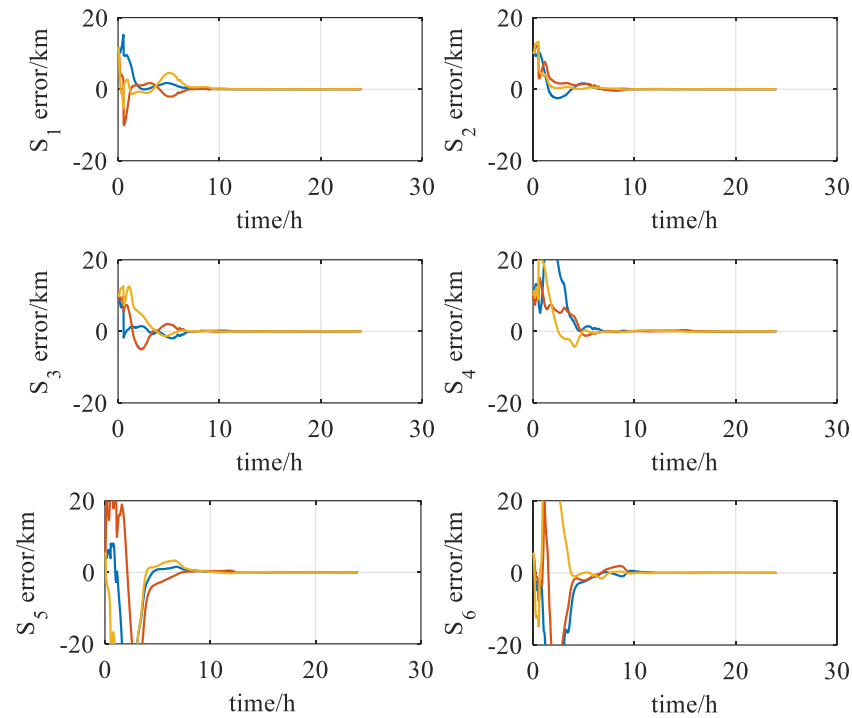

(d) Fully decentralized navigation method

Fig. 8 Orbit determination errors when each spacecraft observes one star using fully decentralized navigation method, integrated navigation method and IREKF

Table 3 Average state uncertainty when each spacecraft observes one star

\begin{tabular}{ccccc}
\hline \multicolumn{2}{c}{ Methods } & IN & FND & IREKF \\
\hline $\mathrm{X} /$ & initial & 10 & 10 & 10 \\
$\mathrm{~km}$ & final & 0.005 & 0.006 & 2.425 \\
$\mathrm{Y} /$ & initial & 10 & 10 & 10 \\
$\mathrm{~km}$ & final & 0.006 & 0.009 & 7.216 \\
$\mathrm{Z} /$ & initial & 10 & 10 & 10 \\
$\mathrm{~km}$ & final & 0.008 & 0.008 & 8.018 \\
\hline
\end{tabular}

\section{Case 3: one spacecraft observes two starlight angles}

In this case, it is assumed that spacecraft $\mathrm{S}_{1}$ observes two different stars and measures two starlight angles. The navigation errors are shown in Fig. 9. The tri-axial state uncertainties are listed in Table 4. In this case, spacecraft $S_{1}$ is capable of determining its absolute orbit independently. The integrated navigation method still has the fastest convergence. The fully decentralized navigation method also realizes the absolute orbit determination, validating the theoretical analysis in theorem 3. The navigation errors of IREKF also converge. But the 
convergence performance from $S_{1}$ to $S_{6}$ gradually decreases. Using IREKF, the orbit of $S_{1}$ is first determined based on the absolute measurements. Then, the orbit of $\mathrm{S}_{2}$ is determined based on the inter-satellite range measurements to $S_{1}$ by referring the orbit of $S_{1}$. Likewise, the orbits of $S_{3}, S_{4}$, $\mathrm{S}_{5}$, and $\mathrm{S}_{6}$ are successively determined. With the growing of the number of spacecraft in a constellation, the convergence performance of the spacecraft which is furthest from spacecraft $\mathrm{S}_{1}$ in the communication crosslink becomes worse. The fully decentralized navigation method overcomes this defect by dividing the absolute orbit determination into two parts. Once the absolute orientation of CBCS is determined, all absolute orbits are determined simultaneously.
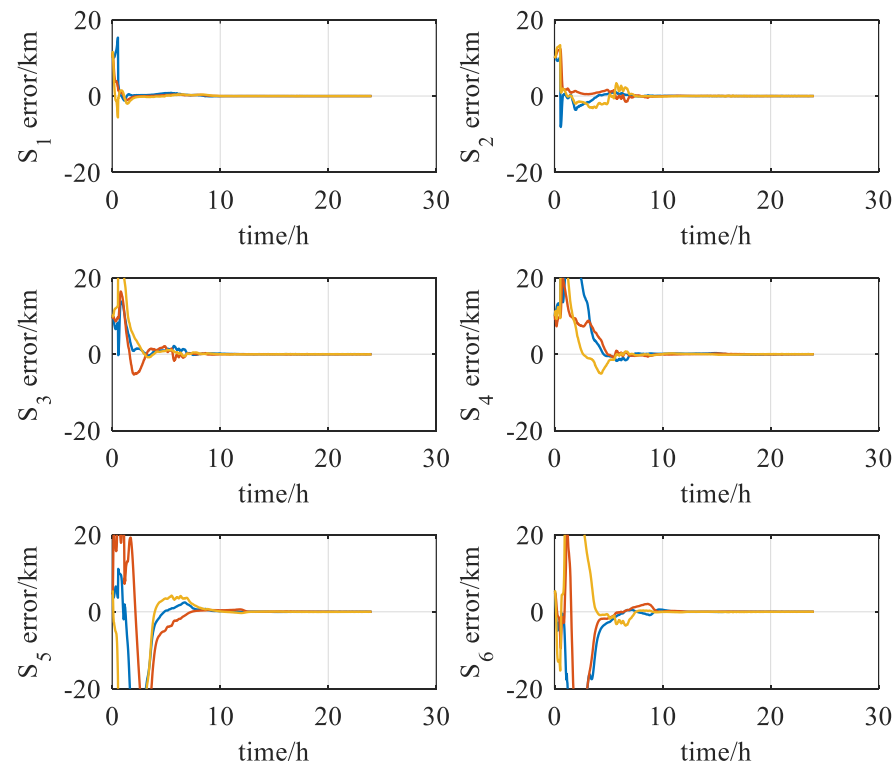

(a) Fully decentralized navigation 

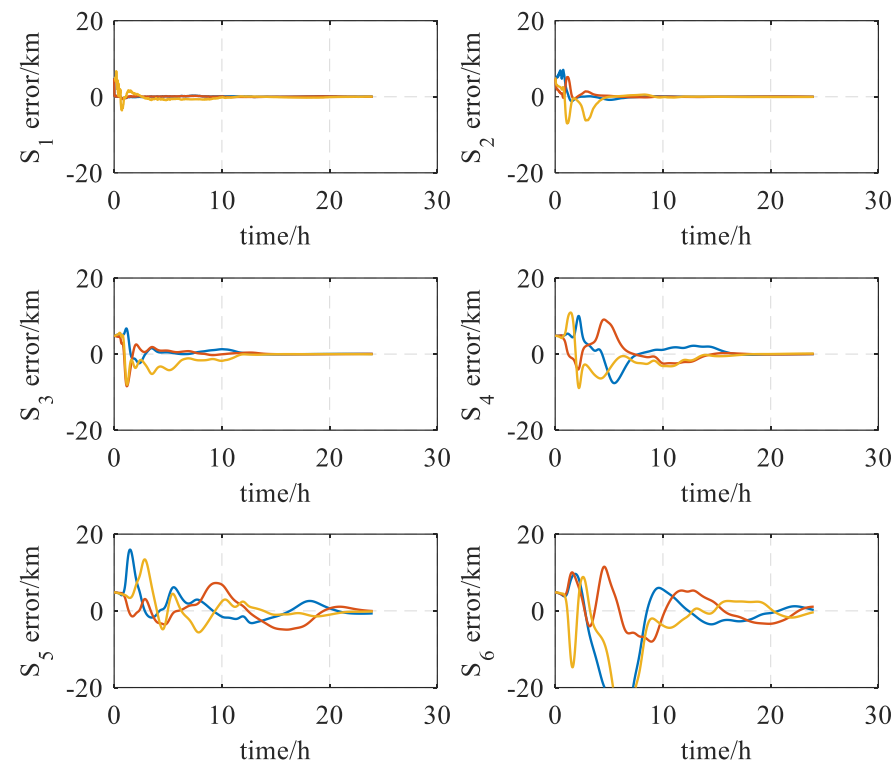

(b) IREKF
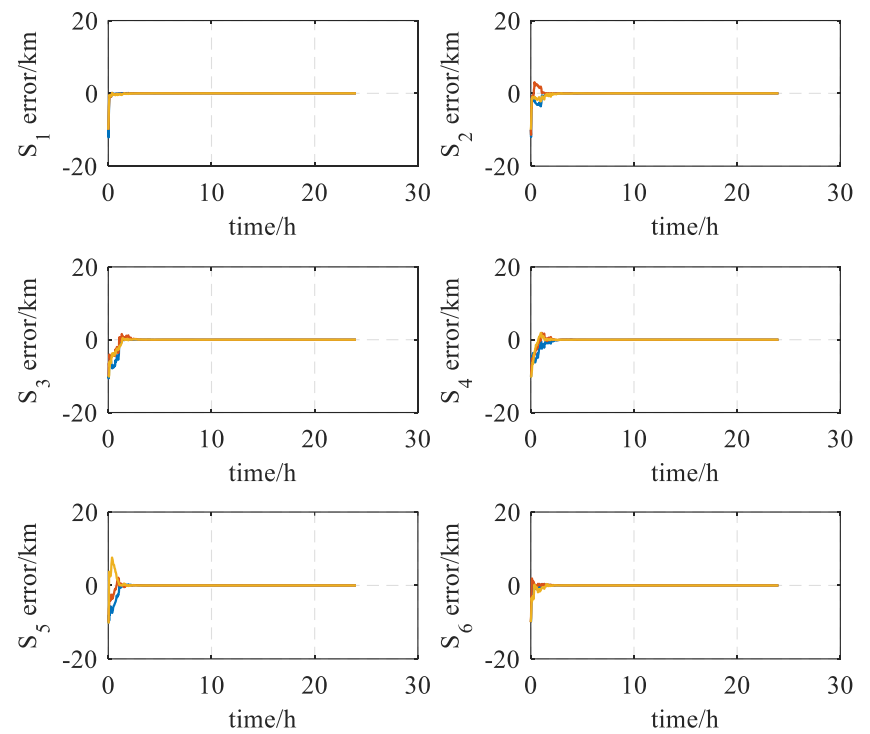

(c) Integrated navigation

Fig. 9 Orbit determination errors when spacecraft $S_{1}$ observes two stars using fully decentralized navigation method, integrated navigation method and IREKF

Table 4 Average state uncertainty when $\mathrm{S}_{1}$ observes two stars

\begin{tabular}{ccccc}
\hline \multicolumn{2}{c}{ Methods } & IN & FND & IREKF \\
\hline $\mathrm{X} /$ & initial & 10 & 10 & 10 \\
$\mathrm{~km}$ & final & 0.007 & 0.009 & 0.045 \\
$\mathrm{Y} /$ & initial & 10 & 10 & 10
\end{tabular}




\begin{tabular}{c|cccc}
$\mathrm{km}$ & final & 0.009 & 0.008 & 0.062 \\
$\mathrm{Z} /$ & initial & 10 & 10 & 10 \\
$\mathrm{~km}$ & final & 0.008 & 0.010 & 0.048 \\
\hline
\end{tabular}

The simulations in the above three cases indicate that the fully decentralized navigation method keeps the following characteristics while distributing the navigation task to each spacecraft. First, the cooperation of spacecraft leads to the fully utilization of measurement information. Measurements from all spacecraft can contribute to absolute orbit determination. Second, the navigation subsystem is equivalent to each other. The convergence performance of each navigation subsystem stays the same. Third, the convergence conditions of the fully decentralized navigation method are the same as integrated navigation.

\section{B. Computation burden}

To reduce the computation burden of the navigation system is the primary purpose of the decentralized navigation. The run time using fully decentralized navigation method when there are two spacecraft is taken as the unit time. The normalized run time of one-step navigation estimation using three navigation methods are shown in Fig. 10. and the specific run time data is listed in Table 5. The simulations are performed in MATLAB language on a computer with an Intel(R) Core i7-8550U CPU @1.8G Hz. It is shown that run time of the integrated navigation grows as a second-order power function with the number of spacecraft. the run time of fully decentralized navigation does not change with the growing of number of spacecraft, neither does IREKF. The state dimension of the fully decentralized navigation is nine, while IREKF is six. Thus, the computation burden of fully decentralized navigation is slightly bigger than the IREKF. 


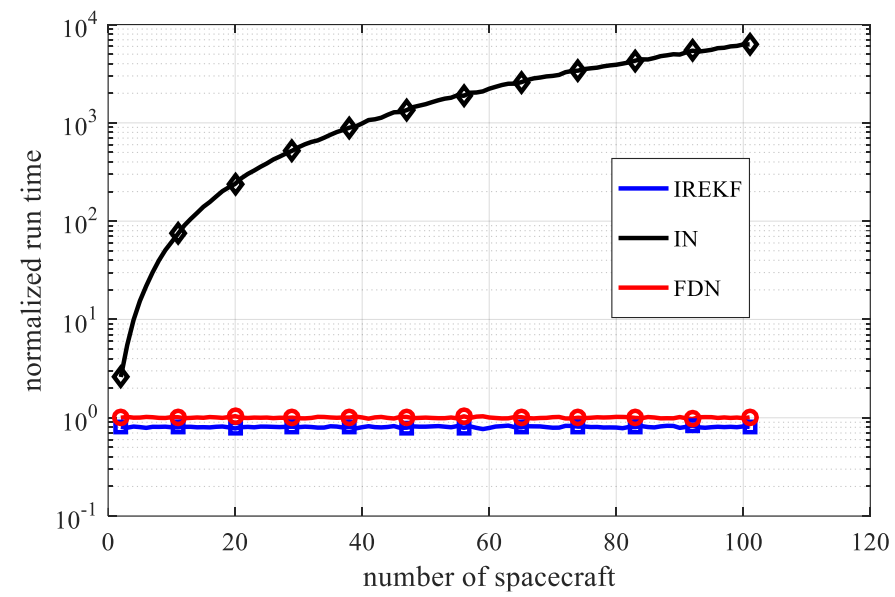

Fig.10 Normalized run time of navigation simulations as the increase of number of spacecraft

Table 5 Run time of one-step estimation using three different methods

\begin{tabular}{ccccccc}
\hline Number of spacecraft & 2 & 40 & 60 & 80 & 100 & $\mathrm{n}$ \\
\hline IN/ms & 36.7 & 14490 & 32421 & 57589 & 90045 & $\sim 9 \mathrm{n}^{2}$ \\
IREKF/ms & 11.7 & 11.6 & 11.7 & 11.8 & 11.6 & $\sim 11.8$ \\
FDN/ms & 14.8 & 14.6 & 14.7 & 14.3 & 14.4 & $\sim 14.4$ \\
\hline
\end{tabular}

\section{Fault Tolerance}

One purpose of the decentralized navigation system is to improve the system reliability, so that the breakdown of a certain subsystem does not affect others. For the integrated navigation system, the breakdown of the main spacecraft would cause failure of the whole navigation system. Consider the case where spacecraft $\mathrm{S}_{1}$ measures two starlight angles. Assume that a fault occurs in spacecraft $S_{3} 24$ hours after the start of navigation, and the states in that navigation subsystem stops updating. The position errors using IREKF and fully decentralized navigation method are shown in Fig. 11. 

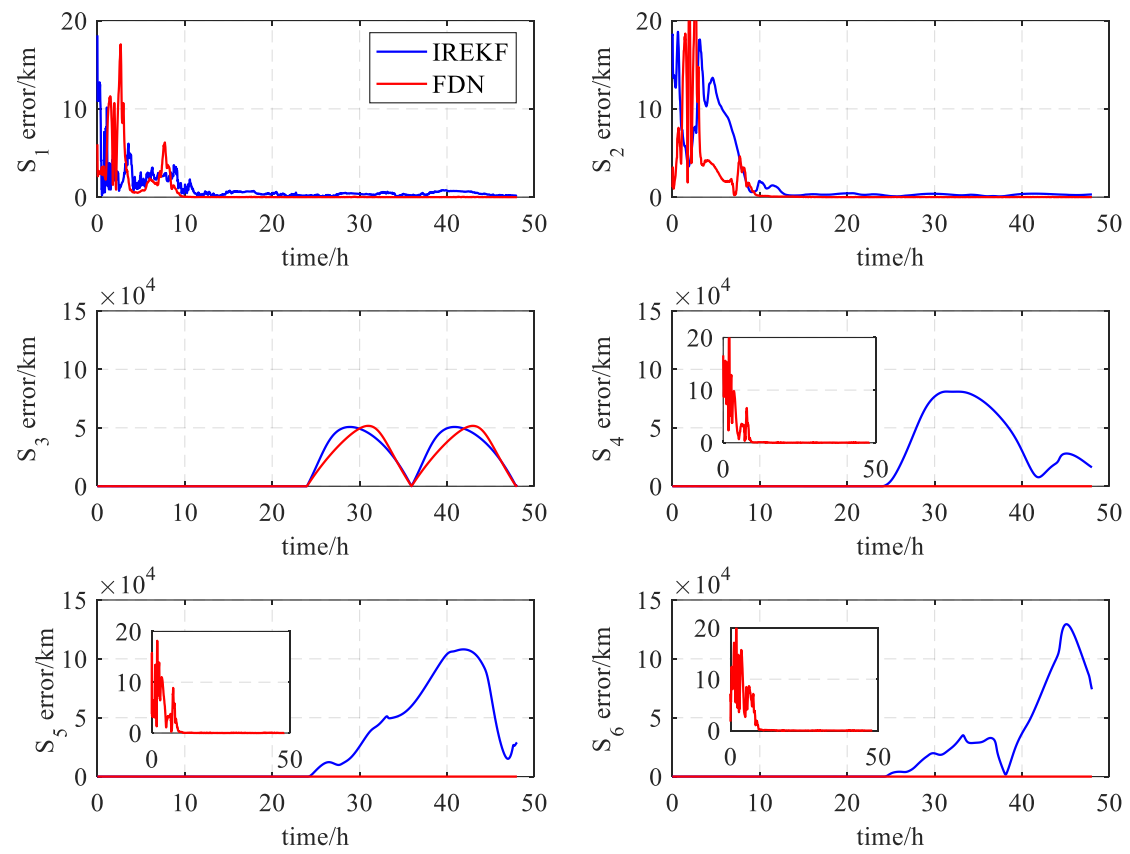

Fig.11 Fault tolerance comparison of two decentralized navigation system.

Using IREKF, the fault in $\mathrm{S}_{3}$ causes no effect on the former spacecraft, while the navigation subsystems on the latter spacecraft all collapse. The nature of IREKF determines that spacecraft $\mathrm{S}_{3}$ is the navigation reference of the latter spacecraft. Using the fully decentralized navigation method, the fault in $S_{3}$ interrupts only the navigation results of $S_{3}$. The navigation subsystems are completely independent with each other. The reliability is further improved compared with IREKF.

\section{Accuracy}

A larger constellation with 30 spacecraft is used for Monte Carlo simulations to analyze the absolute orbit determination accuracy. The orbit elements are shown in Table 6. Assume that spacecraft $S_{1}$ observes two starlight angles with an accuracy of 5 second of arc. The inter-satellite range measurement accuracy is 10 meters. 200-case simulations are performed, and the position errors of the 30-spacecraft in each simulation case are recorded. Fig. 12 shows the average 3-sigma error bounds of the 30 spacecraft. 
Table 6 Orbit elements of a 30 -spacecraft constellation

\begin{tabular}{ccccccc}
\hline \hline Orbit elements & $\alpha / \mathrm{km}$ & $e$ & $\alpha / \mathrm{deg}$ & $\omega / \mathrm{deg}$ & $\Omega / \mathrm{deg}$ & $n / \mathrm{deg}$ \\
\hline $\mathrm{S}_{i}(\mathrm{i}=1,2, \ldots, 30)$ & 26560 & 0.3 & 60 & 10 & $12 i$ & $12 i$ \\
\hline
\end{tabular}

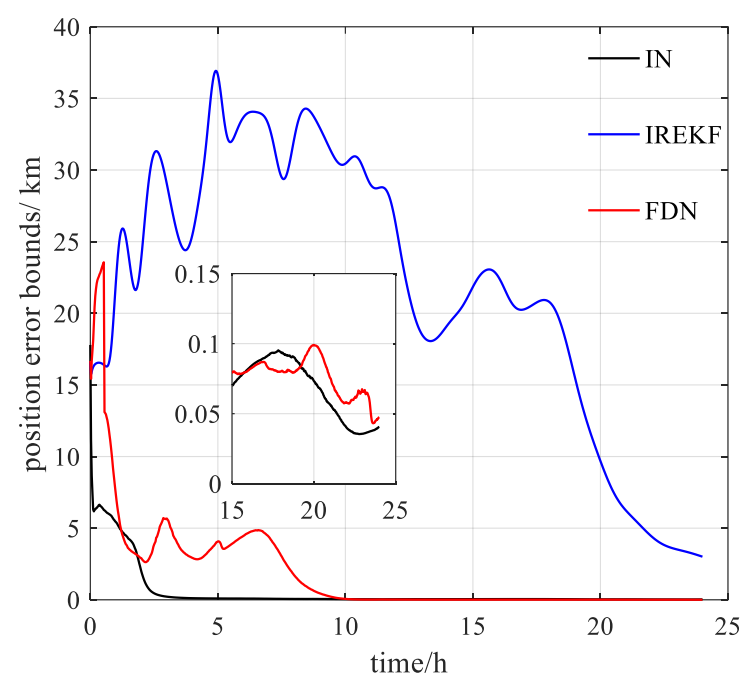

Fig. 12 Average 3-sigma position error bounds of 30 spacecraft in a 200-case Monte Carlo simulations

The errors in the last nine hours are magnified and depicted in sub-windows. The orbit errors using integrated navigation method, IREKF, and fully decentralized navigation method are compared in Fig. 12. It is shown that orbit errors using three methods all converge, illustrating that both approaches can effectively obtain the absolute orbits of all spacecraft. The average 3-sigma initial position error of the 30 spacecraft is $17 \mathrm{~km}$. Using integrated navigation method, the 3 sigma absolute orbit determination accuracy after $24 \mathrm{~h}$ is $40 \mathrm{~m}$. The absolute orbit determination accuracy using fully decentralized navigation method is very close to that using the integrated navigation method, and much higher than the IREKF.

\section{CONCLUSION}

The absolute orbit determination of a spacecraft constellation can be divided into two procedures, relative orbit determination and absolute orientation determination. The relative orbit determination based on inter-satellite range measurements obtains the orbits of all spacecraft in 
the constellation body reference system. The absolute orientation determination, based on starlight angles, obtains the transformation matrix from the constellation body coordinate system to the inertial coordinate system. Then the absolute orbits can be obtained by transforming the orbits from the constellation body coordinate system to the inertial coordinate system. In order to determine the orientation of the constellation, at least two stars need to be observed. In the fully decentralized navigation system, each spacecraft operates a navigation subsystem that only determines the relative orbit between itself and its neighbor spacecraft. Thus, the state dimension of each navigation subsystem stays unchanged with the growing number of spacecraft. Numerical simulations illustrate that the proposed method has a lower computation burden compared with integrated navigation system, and higher accuracy and reliability compared with the conventional decentralized estimation method.

\section{ACKNOWLEDGEMENTS}

This paper is sponsored by the National Natural Science Fund (grant nos. 51827806)

\section{REFERENCES}

[1] Inamiya, K., "Positioning Satellite System Using Intersatellite Communication," Journal of Spacecraft and Rockets, Vol. 28, No. 6, 1991, pp.720-727.

Doi: $10.2514 / 3.26305$

[2] Davis, B. T, Gunter, B. C. "Improving the Collective Precision Orbit Determination of Constellation Satellites Using Ancillary Intersatellite Ranging Data”. AIAA/AAS Astrodynamics Specialist Conference, 2016.

Doi: $10.2514 / 6.2016-5368$

[3] Wang, S., Cui, P., Gao, A., Yu, Z., and Cao, M., “Absolute Navigation for Mars Final Approach Using Relative Measurements of X-ray Pulsars and Mars orbiter', Acta Astronautica, Vol. 138, 
2017, pp.68-78.

Doi: $10.1016 /$ j.actaastro.2017.05.017

[4] Mauro, G. D., Lawn, M., and Bevilacqua, R., "Survey on Guidance Navigation and Control Requirements for Spacecraft Formation-Flying Missions," Journal of Guidance, Control, and Dynamics, Vol. 41, No. 2, 2017, pp. 1-22.

Doi: $10.2514 / 1 . G 002868$

[5] Hu, Y., Sharf, I,. Chen, L., "Three-spacecraft Autonomous Orbit Determination and Observability Analysis with Inertial Angles-only Measurements," Acta Astronautica, Vol. 170, 2020, pp. 106-121.

Doi: $10.1109 /$ ChiCC.2016.7554179

[6] Gnam, C. R., Dianetti, A. D., Crassidis, J. L., "Orbit Determination Using Line-of-Sight and Range Measurements Between Multiple Spacecraft," AIAA Scitech 2020 Forum, 6-10 January 2020, Orlando, FL.

Doi: $10.2514 / 6.2020-1698$

[7] Maisonobe L, Parraud P, Journot M, and Alcarraz-Garcia A. "Multi-satellites Precise Orbit Determination, An Adaptable Open-Source Implementation". 2018 AIAA SpaceOps conference, Marseille, France, 28 May-1 June 2018.

Doi: $10.2514 / 6.2018-2622$

[8] H. Mu, T. Bailey, P. Thompson and H. Durrant-Whyte, "Decentralised Solutions to the Cooperative Multi-Platform Navigation Problem," IEEE Transactions on Aerospace and Electronic Systems, vol. 47, no. 2, pp. 1433-1449, April 2011, Doi: 10.1109/TAES.2011.5751268. 
[9] Widnall, W. S., Gobbini, G. F., "Stability of the Decentralized Estimation in the JTIDS Relative Navigation," IEEE Transactions on Aerospace and Electronic Systems, Vol. 19, No. 2, 1983, pp. $240-249$.

Doi: 10.1109/TAES.1983.309443.

[10] Park, C., "Precise Relative Navigation Using Augmented CDGPS," Doctoral thesis, Department of Mechanical Engineering, Standford University, 2001.

[11] Buse, F., and How, J., "Decentralized Relative Navigation for Formation Flying Spacecraft Using Augmented CDGPS," AIAA Guidance, Navigation, and Control Conference and Exhibit, 5-8 August 2002, Monterey, California.

[12] Ferguson, P., and How, J., "Decentralized Estimation Algorithms for Formation Flying Spacecraft," AIAA Guidance, Navigation, and Control Conference and Exhibit,11-14 August, 2003, Austin, Texas.

Doi: $10.2514 / 6.2003-5442$

[13] Mandic, M., Breger, L., and How, J. P., “Analysis of Decentralized Estimation Filters for Formation Flying Spacecraft," AIAA Guidance, Navigation, and Control Conference and Exhibit, 16 - 19 August 2004, Providence, Rhode Island.

Doi: $10.2514 / 6.2003-5442$

[14] Wang, X., Qin, W., Bai, Y., et al. “A Novel Decentralized Relative Navigation Algorithm for Spacecraft Formation Flying," Aerospace Science and Technology, Vol. 48, 2016, pp. 28-36. Doi: $10.1016 /$ j.ast.2015.10.014

[15] Saini, V., Paranjape, A., and Maity, A., "Decentralized Information Filter with Noncommon States," Journal of Guidance, Control, and Dynamics. Vol. 42, No.9, 2019, pp. 2042-2054. Doi:. 10.2514/1.G003862 
[16] Roy, S., Iltis, R. A., "Decentralized linear estimation in correlated measurement noise," IEEE Transactions on Aerospace and Electronic Systems, Vol. 27, No. 6, 1991, pp. 939-941.

Doi: 10.1109/7.104265.

[17] Kerr, T., "Decentralized Filtering and Redundancy Management for Multisensor Navigation," IEEE Transactions on Aerospace and Electronic Systems, Vol. 23, No. 1, 1987, pp. 83-119. Doi: 10.1109/TAES.1987.313339.

[18] Di Mauro, G., Lawn, M., and Bevilacqua, R., "Survey on Guidance Navigation and Control Requirements for Spacecraft Formation-Flying Missions," Journal of Guidance Control and Dynamics, Vol. 41, No. 3, 2017, pp. 1-22.

Doi: $10.2514 / 1 . G 002868$

[19] Wang, J., Butcher, E. A., "Decentralized Estimation of Spacecraft Relative Motion Using Consensus Extended Kalman Filter,” 2018 Space Flight Mechanics Meeting, AIAA SciTech Forum, AIAA Paper 2018-1965, 2018. Doi:10.2514/6.2018-1965

[20] Qin, T., Qiao, D., MacDonald, M., "Relative Orbit Determination Using only Intersatellite Range Measurements," Journal of Guidance, Control, and Dynamics, Vol. 42, No. 3, 2019, pp. 703-710.

Doi: $10.2514 / 1 . G 003819$

[21] Jiang, J., Wang, H., Zhang, G. "High-Accuracy Synchronous Extraction Algorithm of Star and Celestial Body Features for Optical Navigation Sensor," IEEE Sensors Journal, 2018, 18(2): 713-723.

Doi: 10.1109/JSEN.2017.2777493.

[22] Zhang, Y., Jiang, J., Zhang, G., et al. "Accurate and Robust Synchronous Extraction Algorithm for Star Centroid and Nearby Celestial Body Edge," IEEE Access, 2019, 7: 126742-126752. 
Doi: 10.1109/ACCESS.2019.2939148. 